\title{
Circuit
}

Musiques contemporaines

\section{Introduction : des formations qui demeurent}

\section{Jonathan Goldman}

Volume 23, numéro 3, 2013

Géométries durables : pour les 25 ans du Nouvel Ensemble Moderne

URI : https://id.erudit.org/iderudit/1021514ar

DOI : https://doi.org/10.7202/1021514ar

Aller au sommaire du numéro

Éditeur(s)

Les Presses de l’Université de Montréal

ISSN

1183-1693 (imprimé)

1488-9692 (numérique)

Découvrir la revue

Citer ce document

Goldman, J. (2013). Introduction : des formations qui demeurent. Circuit, 23(3),

5-8. https://doi.org/10.7202/1021514ar d'utilisation que vous pouvez consulter en ligne.

https://apropos.erudit.org/fr/usagers/politique-dutilisation/ 


\section{Introduction : des formations qui demeurent}

Jonathan Goldman

Un article récent d'Eric Drott trace l'histoire d'un certain récit de la musique du XXe siècle'. Selon cette intrigue, multipliée dans d'innombrables manuels d'école, l'histoire de la musique du siècle passé serait celle d'un affranchissement progressif de l'œuvre des contraintes liées au genre. L'œuvre cesse d'être sonate, symphonie, bourrée ou menuet pour devenir sui generis - chaque œuvre définissant un genre dont elle est le seul représentant. Difficile de nier la force de ce récit, lorsque, par exemple, dans son Esthétique de la musique (1967), Carl Dahlhaus affirme qu'alors que, dans la musique du passé, une œuvre fut d'abord un exemple d'un genre, «in the twentieth century, individual structures submit only under duress to being allocated to any genre ${ }^{2}$ ». Néanmoins, Drott nous permet d'entendre une dissonance entre ce récit convenu et la réalité de la musique du $\mathrm{XX}^{\mathrm{e}}$ siècle, où certains genres perdurent sans se soucier des avis de décès. Pour illustrer sa thèse, Drott se penche sur les Espaces acoustiques (1974-1985) de Gérard Grisey et trouve dans ce cycle du chef de file du courant spectral des contextes génériques (par exemple, la musique classique, la musique contemporaine européenne, la musique spectrale, etc.) qui induisent autant d'écoutes différentes.

Ce numéro sera donc une exploration de genres ou de formations instrumentales qui persistent jusqu'à l'époque actuelle, malgré toutes les critiques qu'une certaine esthétique moderniste a pu diriger à leur encontre. Je dis bien genres ou formations, car il demeure en musique classique une ambiguïté, toutefois féconde, entre le genre au sens de la forme d'une œuvre et au sens de son instrumentation. Une «sonate pour piano » désigne à la fois l'instrumentation et la forme générale (même si, à la lecture des Formes sonate - au titre significativement pluriel - du regretté Charles Rosen, nous avons l'impression d'y trouver plus d'exceptions que de règles $\left.{ }^{3}\right)$. Mais ce
1. Eric Drott (2013), "The End(s) of Genre", Journal of Music Theory, vol. 57, $\mathrm{n}^{\circ} 1$, p. 1-45.

2. Carl Dahlhaus (1982), Esthetics of Music, translated by William Austin, Cambridge, Cambridge University Press, p. 15; cité in Drott, 2013, p. 2.

3. Charles Rosen (1993), Formes sonate, Arles, Actes-Sud. 
4. Dans le documentaire produit par le réseau PBS, Great Performances: Steve Reich - A New Musical Language (1987). Je remercie le compositeur Anthony Genge de m'avoir indiqué cette référence.

5. Sur le site du compositeur: $<w w w$. daifujikura.com/un/lw_PC.html> (consulté le 11 octobre 2013).

6. Voir Pierre Boudrieu (1977), "La production de la croyance", Actes de la recherche en sciences sociales, vol. 13, $\mathrm{n}^{0}$ 1, p. 3-43.

7. Voir le site du compositeur: $<$ www.pernoergaard.dk> (consulté le 11 octobre 2013). n'est pas seulement le cas de la sonate, ce métagenre de la musique classicoromantique. En effet, presque toutes les autres désignations génériques sont également concernées: le trio avec piano, le quatuor à cordes, la symphonie, etc. Cette ambiguïté, loin de s'estomper au $\mathrm{XX}^{\mathrm{e}}$ siècle, s'accentue au fur et à mesure que les titres et les sons réverbèrent au rythme d'une sensation de rupture et d'éloignement des sources de la tradition.

Ainsi, malgré les critiques, le genre perdure non seulement au travers des musicologues de poids comme Dahlhaus, mais surtout par le biais des compositeurs. Certains d'entre eux renoncent aux genres jugés surannés, tandis que d'autres cessent d'évoquer le genre dans leurs titres d'œuvres, leurs notices ou leurs entretiens publics, mais continuent d'y faire appel dans leur musique. Cela fait bien longtemps que je souhaitais rassembler toutes les notices de concert, rédigées pour accompagner des œuvres pour instrument soliste et ensemble, où le compositeur se presse d'affirmer qu' «il ne s'agit pas d'un concerto au sens classique du terme ", qu'il ne faut pas s'attendre à « une opposition entre soliste et orchestre, comme c'est le cas dans le concerto classique », ou autre caveat emptor du «genre ». Prenons deux exemples. Dans un film documentaire, Steve Reich affirmait qu'après que le flûtiste Ransom Wilson lui a proposé une commande d'un concerto pour flute, il lui avait répondu: «"no", because I don't like the idea of concertos, I don't like the idea of the soloist pitted against the orchestra. And, I also don't feel that way in the musical sociology of things, I prefer the ensemble rather than the soloist ${ }^{4}$.» Pour sa part, le compositeur Dai Fujikura a décrit la composition de son concerto Ampere (2008), pour piano et orchestre, comme un «cauchemar »: «I really don't like musical material to be shared between the piano and the orchestra. I don't write in a Romantic idiom - which lends itself to the concerto form - with one solo instrument duelling with an orchestra over the same melod $y^{5}$.» Nous avons affaire ici, me semble-t-il, à un locus classicus de la « dénégation », que Pierre Bourdieu a su analyser dans le fonctionnement du discours du galeriste de l'art contemporain 6 .

Rares sont les compositeurs qui assument, comme le fait Per Nørgård à propos de son Concerto en due tempi (1996) au sens classique du terme », comme il est plus ou moins le cas même pour les œuvres que l'on ne taxerait pas de conservatisme comme la série des Chemins de Luciano Berio ou celle des Trames de Martin Matalon. C'est pourquoi je suis particulièrement ravi d'accueillir un article de John Rea qui propose une réflexion sur le concerto au XXe siècle. L'auteur est par ailleurs lui-même le compositeur d'un non-concerto, les Figures hâtives, œuvre pour violon solo (Richard Roberts) et orchestre créée par l'Orchestre symphonique 
de Montréal en 2006 (Yoav Talmi, direction). Ne nous étonnons donc pas si l'article - le mot ne convient pas à un texte qui renvoie clairement à plusieurs genres dramatico-littéraires - se présente sous forme d'un dialogue exempt d'opposition, au sens classique du terme...

En ce qui a trait à la musique contemporaine, le chef de file de ces genres/formations qui se perpétuent se trouve bien sûr dans l'œuvre pour ensemble de musique contemporaine. Les adeptes des concerts du Nouvel Ensemble Moderne (NEM) à Montréal, de l'Ensemble intercontemporain à Paris, de l'Ensemble Dal Niente à Chicago ou de l'Ensemble Modern à Francfort, sont habitués à des œuvres que je m'amuse parfois à appeler le «15/15»: des œuvres de 15 minutes pour 15 instruments, genre typique, souvent imposé dans des concours de composition. La persistance d'œuvres composées pour de tels effectifs instrumentaux s'explique bien sûr en partie par le dynamisme de ces ensembles qui commandent des œuvres afin d'étoffer leur répertoire. Cependant, des raisons plus profondes ne sont pas à exclure. L'œuvre pour ensemble de musique contemporaine, cette symphonie de chambre qui trouve ses antécédents dans des œuvres du début du XXe siècle pour effectifs réduits comme la Symphonie de chambre (1906), op. 9, d'Arnold Schoenberg, est devenue un classique aux XXe et XXI ${ }^{\mathrm{e}}$ siècles, et constitue sans doute un genre, pourvu qu'elle crée une série d'attentes chez l'auditeur.

Cela m'amène au point de départ de ce numéro : commémorer les 25 ans de l'ensemble de musique contemporaine par excellence, le NEM, dirigé par Lorraine Vaillancourt. Cet ensemble a été fondé au même moment et dans un seul geste avec la revue Circuit, et je suis ravi de marquer ce grand moment, le quart de siècle étant un cap appréciable. La revue célèbre l'événement par un dossier sur le NEM qui prolonge le dossier thématique de ce numéro et comprend trois étapes nous initiant aux réalisations immenses de cet ensemble: un entretien avec Lorraine Vaillancourt réalisé par mes soins, une visite guidée dans les archives du NEM par Ariane Couture et une discographie commentée par Cléo Palacio-Quintin. Le dossier est complété par un supplément web, le film documentaire réalisé en 2001 par James Dormeyer sur le NEM, mettant en scène la création de Menuhin: Présence, œuvre ultime et profonde du compositeur André Prévost (1934-2001) pour violoncelle et le NEM, et offrant, entre autres merveilles, un aperçu sur le fonctionnement des répétitions de l'ensemble.

Outre le «genre» de ce que j'ai nommé le «15/15», il existe d'innombrables géométries non pas variables mais durables au sein de la musique contemporaine, si l'on pense à l'ensemble Pierrot - soit l'instrumentation que Schoenberg a choisie pour Pierrot lunaire $(1912)^{8}$-, le quatuor dit «pour
8. Voir Christopher Dromey (2013) The Pierrot Ensembles: Chronicle and Catalogue, 1912-2012, edited by Christopher Wintle, Rochester, Boydell and Brewer. 
la fin du temps» - qui comprend un violon, une clarinette, un piano et un violoncelle, instrumentation de la fameuse œuvre d'Olivier Messiaen - ou le sextuor «Dérive»- pour flûte, clarinette, violon, violoncelle, piano et percussion, d'après l'œuvre Dérive 1 (1984) de Pierre Boulez. Nous pourrions prolonger cette liste à l'infini avec le monodrame avec trame électronique ou le cycle pour instrument solo, mais l'un des genres qui a le mieux traversé le temps est certainement celui du quatuor à cordes, une source d'innovation apparemment inépuisable chez les compositeurs des 250 dernières années. C'est pourquoi nous avons invité Clemens Merkel, violoniste et cofondateur du Quatuor Bozzini, à penser ce genre/formation du point de vue de l'interprète. Enfin, dans l'enquête de ce numéro, Jimmie LeBlanc interroge sept acteurs du milieu musical montréalais sur leur rapport aux géométries durables.

Pour terminer, la traditionnelle rubrique des Actualités vient conclure ce dernier numéro du volume 23 de Circuit. Tout d'abord, Maxime McKinley nous présente un compte rendu d'une conférence du compositeur Gérard Pesson à propos de sa collaboration avec le poète Philippe Beck, donnée le 30 août dernier à l'occasion du colloque Philippe Beck, un chant objectif aujourd'hui, organisé à Cerisy du 26 août au 2 septembre 2013. S'ensuit la chronique "Créé dans LeVivier », rédigée par Cléo Palacio-Quintin, qui nous propose une rétrospective des créations de la saison artistique 2012-2013 par les organismes musicaux partenaires du Vivier.

Bonne lecture!

Montréal, octobre 2013 